\title{
The Role of Vidura Chatbot in the Diffusion of KnowCOVID-19 Gateway
}

\author{
Kerk F. Kee ${ }^{1}$, Prasad Calyam ${ }^{2}$, and Hariharan Regunath ${ }^{3(\mathbb{D}}$ \\ 1 College of Media \& Communication, Texas Tech University, Lubbock, TX, USA \\ 2 Department of Electrical Engineering and Computer Science, University of Missouri-Columbia \\ 3 Department of Medicine-Divisions of Pulmonary, Critical Care Medicine and Infectious Diseases, \\ University of Missouri-Columbia
}

\section{Abstract}

The COVID-19 pandemic is an unprecedented global emergency. Clinicians and medical researchers are suddenly thrown into a situation where they need to keep up with the latest and best evidence for decision-making at work in order to save lives and develop solutions for COVID-19 treatments and preventions. However, a challenge is the overwhelming numbers of online publications with a wide range of quality. We explain a science gateway platform designed to help users to filter the overwhelming amount of literature efficiently (with speed) and effectively (with quality), to find answers to their scientific questions. It is equipped with a chatbot to assist users to overcome infodemic, low usability, and high learning curve. We argue that human-machine communication via a chatbot play a critical role in enabling the diffusion of innovations.

Keywords: COVID-19, science gateways, chatbot, human-machine communication, diffusion of innovations

\section{Introduction}

The COVID-19 pandemic is one of the world's greatest crises in the new millennium. As of May 2021, about 163 million people worldwide contracted the SARS-CoV-2 coronavirus, and 3.38 million people sadly lost their lives to this disease (WHO, 2021). Furthermore,

CONTACT Kerk F. Kee (D) - College of Media \& Communication • Texas Tech University • Box 43082 - Lubbock, TX 79409 • kerk.kee@ttu.edu 
it is estimated that the pandemic led to a USD 3.94 trillion loss in economic output globally during 2020, approximately a $4.5 \%$ drop in gross domestic product (GDP) (Szmigiera, 2021). The unprecedented impacts will have lasting effects for many years. During the pandemic, many new ideas have been implemented to address the various problems that stemmed from the pandemic, including in the areas of infection prevention and control, public health measures, and so forth. As the old saying goes, "Necessity is the mother of invention." New technologies, practices, behaviors, approaches, and so forth, are often developed as solutions to difficult problems people face.

Chatbot can be used for many creative solutions for problems during the pandemic (Mehfooz et al., 2021). For example, one of the major problems during the pandemic lies in the testing or screening of patients for COVID. However, the volumes are overwhelming, and health care workers are at risk of being exposed to the virus during the testing/ screening process. This is where machines (e.g., chatbots) can help. In addition to working with patients with non-direct contacts and reducing the risks on health care workers, machines can work tirelessly to address the rapid surge in cases, and can also adapt to multiple languages (Martin et al., 2020). Human-machine communication (HMC) between a multilingual chatbot and patients around the world can improve both the effectiveness (with the latest COVID-19 information) and efficiency (with around-the-clock communication with patients) of addressing the pandemic. We define HMC as the communication between humans and machines in accomplishing the common goals of identifying the best care possible for patients and the best research evidence for treatment and prevention strategies. Therefore, HMC plays a crucial role in the global efforts in combating COVID-19 during the pandemic.

Researchers have experimented with chatbots to answer questions about COVID-19 (VolppKevin, 2020). In Thailand, a chatbot was designed to answer questions about topics such as how to protect oneself from coronavirus, self-screening for symptoms, a list of nearest hospitals, fake news about COVID-19, and so forth (Rodsawang et al., 2020). During the pandemic, patients cannot easily see their primary care doctors in person for regular non-COVID cases. In India, Medbot was created to be a chatbot that can provide telemedicine in place of a doctor, providing advice and tips on general preventive measures, checking symptoms, suggesting home remedies, and providing counseling for general well-being (Bharti et al., 2020). Chatbots have also been used to perform tele-psychiatry for patients suffering from pathological social withdrawal, intensified by the stay-at-home orders, lockdowns, and social distancing (Yoshikawa et al., 2021). Beyond health care applications (Ling \& Björling, 2020), chatbots have also been incorporated into crisis reporting and news platforms, to help deliver timely information about COVID-19 to the public (Maniou \& Veglis, 2020).

Innovations are needed for filtering the surplus scientific literature on COVID-19 so that clinicians and medical researchers can efficiently seek and learn from the most recent and relevant publications (Oruche et al., 2021). This article seeks to explore the research question: "How can an AI-powered chatbot help users, especially clinicians and medical researchers, effectively and efficiently identify reliable information with high evidence levels to help them make the best decisions at work?" Answers to this question can shed light on how HMC contributes to the diffusion of innovations (Rogers, 2003) for combating the COVID19 pandemic in the present moment. Furthermore, answers to this question can help us 
understand how HMC contributes to the new normal in the post-pandemic world as well as how HMC can better prepare us for the next pandemic or global emergency, where similar challenges such as those related to the current pandemic re-emerge in the future.

HMC research on machine's advice-giving suggests that the difficulty of the tasks and perceived machine's understanding of decision consequences matter in human users' advice utilization (Prahl \& Van Swol, 2021). Given the low usability and high technicality of many gateways as well as the scientific and societal significance of having the latest and best COVID-19 literature for clinicians and medical researchers in combating the pandemic, a study on an advice-giving chatbot can help advance HMC research in designing machines that will enjoy a higher degree of acceptance, adoption, and utilization by users. Although some HMC scholars are concerned about negative effects of machines replacing humans at the workplace (Gibbs et al., 2021; Piercy \& Gist-Mackey, 2021) and raised ethical questions in critical studies on HMC (Dehnert \& Leach, 2021; Liu, 2021), the potential positive consequences of a machine-driven solution can help clinicians and medical researchers save lives, a task that Prahl and Van Swol (2021) would term humanitarian. Hence, a machine-driven solution may be well-received in HMC research.

In this article, we explain a science gateway platform called KnowCOVID-19 (Oruche et al., 2021), equipped with a chatbot called Vidura (Chandrashekara et al., 2018). KnowCOVID-19 is designed to automatically sort scientific literature based on different types of evidence (e.g., empirical studies versus expert opinions). Named after a wise advisor in Indian mythology, Vidura is a chatbot designed to guide users to navigate the platform to effectively (with quality) and efficiently (with speed) find the scientific literature with the desired type of evidence to help clinicians and medical researchers combat the pandemic, including giving the best care to patients with COVID-19 as well as discovering treatments and preventions for the disease. Both the gateway and the chatbot are in the prototype phase under a collaborative project funded by the National Science Foundation in the United States (see Calyam \& Nair, 2020; Kee, 2020).

Given the urgent need to develop a chatbot to leverage HMC to combat the COVID-19 pandemic, this article will first define the machine (i.e., a science gateway integrated with an AI-powered chatbot) central to the core of this article. Second, we elaborate on the main problems faced by users in our specific context of scientific literature searches on COVID19. Third, we explain how three novel solutions can be integrated into technologies to help overcome the stated problems. Fourth, we briefly describe the functions of a gateway platform and a chatbot to help users with optimizing scientific literature searches on COVID19. Fifth, we propose how the adoption of our chatbot can be further studied, using a mix of methods commonly used in communication and HMC research. Finally, this article concludes by providing some theoretical and practical implications of the role of chatbot and $\mathrm{HMC}$ in diffusion research.

\section{AI-Powered Chatbot Integrated With a Science Gateway for COVID-19}

It is important to discuss the concepts of artificial intelligence (AI) and chatbot. AI involves "the study of agents that exist in an environment and perceive and act" (Russell \& Norvig, 1995, p. 5). AI is not simply a computer program, but a smart computer program (often 
referred to as a computer agent) that can simulate human thoughts and actions toward optimal outcomes according to specified goals. AI-driven machines are often said to mimic the human minds in order to learn and solve problems at a higher scale and with a higher reliability that is not feasible manually.

AI involves machine learning (ML) and deep learning (DL). ML is a technique that allows computers to improve its performance based on inputs and experiences. On the other hand, DL is a technique to program computers to learn, modeling after human multilayer neuro networks. Given these techniques, AI-powered machines can augment human functions at large scales through automation and further improve their performance through recommendation of a set of viable options for humans to consider to process data. In this article, AI in the form of topic models of data and recommender engines to provision computational resources provides much of the foundation of how our Vidura chatbot operates in improving the effectiveness and efficiency of KnowCOVID-19 science gateway to filter huge publication archives of information.

Chatbot, one of the most prevalent practical AI examples, is a conversational agent that imitates human-to-human conversations. There are text-based chatbots (e.g., Bank of America's Erica, Capital One's Eno, Geico's Kate, Amtrak's Julie, etc.) (Ali, 2021) and voicebased chatbots (e.g., Amazon Alexa, Apple Siri, Google Assistant, Microsoft Cortana, etc.) (Etzrodt \& Engesser, 2021). As alluded to in the previous paragraph, although users directly interact with the chatbot such as Erica and Alexa, the AI algorithms form an integral part of the users' experience with the chatbot. Therefore, the concepts of AI and chatbot can be treated as one, as the chatbot cannot function properly without AI, and the AI is not usable without a well-designed chatbot user interface.

More recently, the bibliometric study on chatbots (Io \& Lee, 2017) reveals the scope of contributions in the state-of-the-art for chatbot applications in many domains with a high number of alternatives and thus justifies the need for (semi-)automated user support. This also confirms the novelty of our contribution to science gateway communities, since, to the best of our knowledge, there is no prior work in this area. In other recent works, a new category of conversational agents with chatbot interfaces enables natural language processing to map suitable commands for their execution to provide convenience for user requirement. An example is implemented in prior work (Bieliauskas \& Schreiber, 2017) for a conversational visualization service to extract keywords from the conversation and to apply filters to the visualizations. Such methods are helpful in data science applications as they allow novice users to build data models with minimal coding. Chatbots for data analysis workflow management such as AVA (John et al., 2017) and more recently IRIS (Fast et al., 2018) are promising solutions that further motivate our work, and the benefits that we expect for the science gateway communities.

A science gateway refers to a "community-specific set of tools, applications, and data collections that are integrated together via a portal or a suite of applications, providing access to grid-integrated resources" (Wilkins-Diehr, 2006, p. 743). Via a web-based graphical user-interface, data providers share their data with a community of users and other providers. On the other hand, users can access shared resources, such as software, instruments, (de-identified) data, computing (cloud) services, educational materials as well as disciplinespecific knowledge as a diverse community of users (SGCI, 2020). In other words, a gateway 
is a point-and-click platform to help users harness and process big data through web services of computational resources.

What is the big data in this article? As previously mentioned, the amount of scientific literature in publication archives such as PubMed (NCBI, 2020), LitCovid (NLM, 2020) being produced on COVID-19 is vast and fast-growing, which has reached an overwhelming state for clinicians and medical researchers. Not only is the data big in size, today's big datasets are often characterized by multiple parameters (Mayer-Schönberger \& Cukier, 2014). As a result, many clinicians and medical researchers are unable to keep up with the volume of information being produced daily. We further elaborate on this problem of data deluge along with two other related challenges in the next section.

\section{The Three Problems of Big Data, Quality, and Diffusion}

In our particular context of scientific literature searches, clinicians and medical researchers face three major problems that we termed the big data problem, the quality problem, and the diffusion problem. First, the big data problem. The amount of literature being produced on COVID-19 is vast and fast-growing, exponentially higher than any other topic in scientific research prior to the pandemic. This is understandable given the urgency of the global emergency. Many researchers across the world are devoting their time and concerted efforts to combat the pandemic. However, the size of the data is so overwhelming that it created a dilemma for clinicians and medical researchers in that they cannot keep up with the literature. Furthermore, the data size makes it difficult for clinicians and medical researchers to find the exact information with the desired level of evidence they need for their individual work in combating COVID-19.

Second, is the quality problem. The traditional peer-review process is long and slow, as a paper needs to undergo multiple rounds of peer reviews and authors' revisions before it can be accepted for publication. This process can sometimes take several months to a couple of years, depending on the journals. Although this process ensures a high level of quality for the publications, COVID-19-related research cannot wait to undergo such a lengthy process. Otherwise, published findings will be outdated and/or miss the opportunity windows to save lives. The need to rush through the traditional peer review process is apparent, given the urgency to share COVID-related medical findings across the world. When the peer review process is rushed, the quality of review is compromised. In many situations, the urgency also pushed researchers to publish their results in the form of pre-prints. Pre-prints are research papers that can be made publicly available online immediately. Furthermore, some of the pre-prints are recommendations based on expert opinions, and not empirical studies. Therefore, the quality of evidence varies widely. This creates the data quality problem, which in turn impacts the data reliability as a clinician performs searches with keywords and then manually sorts through a wide range of literature with varying quality to make decisions that are critical for patient care.

Third, the diffusion problem. Although science gateways are designed with a webbased user-interface with the goal of removing the need for command-line programming to harness big data, individual adoption and systemic diffusion is still heavily compromised (Kee et al., 2021). This is understandable given that gateways are built for specific purposes, 
and many of them suffer from a low level of usability when more functionality is added over time. Many of the prototype gateways were developed by lead-users who cannot find off-the-shelf solutions to their big data problems in scientific research. Therefore, these prototype gateways were developed for their own use and the use of a small initial group of peers and collaborators who may not be concerned about usability (Kee et al., 2016). However, the lead users' willingness to tolerate a low level of usability can be a big barrier to mass adoption beyond the initial small group or when additional functionality needs to be added or enhanced. A related issue is the lack of technical support, especially 24-hour around-the-clock and 7 days a week (Kee et al., 2021). The issue is further compounded by the fact that user support is time-intensive, often requiring one-on-one help and training (Nair \& Calyam, 2018). Therefore, while gateways are a promising solution, the adoption and diffusion are challenged by the limitations in usability and user support. In this particular article, the diffusion problem refers to the need for users to quickly learn how to use the KnowCOVID-19 gateway and to receive technical support, such that the adoption and diffusion of this gateway innovation can be sustained over time to combat the pandemic.

These three problems of big data, quality, and diffusion present the challenges we seek to overcome with a gateway KnowCOVID-19. By leveraging Vidura, an AI-powered chatbot, to assist users to conduct literature searches, clinicians and medical researchers can improve the quality of the information they find. The next section explains how KnowCOVID-19 and Vidura, the gateway and chatbot, help overcome the three stated problems.

\section{Gateways, Levels of Evidence, and Chatbots as Solutions to the Three Problems}

To address the big data problem, science gateways have been developed in different domains, such as neuroscience, physics, chemistry, and material science (Vekaria et al., 2020). Gateways have also integrated smart devices to provide smart solutions for health care professionals and patients (Chandrashekara et al., 2018). In our case, clinicians and medical researchers can access a wide scope of online literature on COVID-19 (text-based data analytics) to help them find the answers to their questions as the starting point. As a computer science technique, topic modeling of existing scientific literature can be leveraged (Zhang et al., 2018), thus sorting literature into topics based on clinical queries and categories based on specific clinical groupings. Furthermore, although it was discussed earlier that an overwhelming volume of literature is a problem, big data also provide advantages over small data. For example, the gateway can include scientific literature on studies of previous coronavirus, such as MERS in 2012-2013 and SARS in 2002-2003. Such an approach can allow users to run correlational and/or associational analyses to identify longer-term patterns in the literature for infectious diseases and display the results and findings via visualizations.

Second, to address the quality problem, we turn to what has become known as the Levels of Evidence (LOE) by Ackley et al. (2008). Briefly, LOE distinguishes the types of evidence used in a paper, assigning them from level VII (the lowest level) if the evidence is based on expert opinions to level 1 (the highest level) if the evidence is based on a systematic review or a meta-analysis of multiple rigorous studies, such as randomized controlled trials. Greenhalgh (2014) expanded on the notion and ranked the different types of evidence based on a hierarchy using a pyramid, listing meta-analysis at the top of the pyramid, 
followed by systematic review, critically appraised topic, critically appraised articles, randomized controlled trials, cohort study, case-control study, background information/expert opinions, and finally animal research/lab studies. LOE provides an objective way to rank the overwhelming number of COVID-related publications and help clinicians and medical researchers filter through the sea of literature to identify the best quality evidence for their practice. Essentially, the gateway is designed to allow users to search and retrieve a large quantity of scientific and medical literature on health care topics related to COVID-19, and then sort the literature based on the pre-determined hierarchy of evidence to help users expedite and optimize their search process to obtain the best results.

Third, to address the diffusion problem, a chatbot assistant is designed to provide user support around the clock. Chatbot assistants have been implemented to provide user support in various industries, such as banking, customer service, and online education (Baby et al., 2017; Muslih et al., 2018; Ni et al., 2017; Zhang et al., 2018). The same idea can be applied to user support for using gateways. By combining the solutions of all three problems, the gateway can remove the burden of manual searches and a high learning curve due to a gateway's low usability and technical difficulty, by leveraging the chatbot assistant powered by $\mathrm{AI}$ in the form of recommender engines, topic models of datasets, and other techniques.

\section{The KnowCOVID-19 Gateway and the Vidura Chatbot}

For the purpose of developing the KnowCOVID-19 gateway, more than 10,000 publications were gathered from the Kaggle COVID-19 Open Research Dataset (CORD-19) (Eren et al., 2020). Based on this dataset, users can perform three general activities with the literature searches. First, using the gateway, users can enter keywords and search terms and identify the levels of evidence they are interested in finding. As previously discussed, the total amount of literature has a wide range of quality. By identifying specific levels of evidence, users can narrow their search results to particular types of evidence they believe to be credible and appropriate for their purpose associated with certain keywords.

Second, users can select the articles from the search results generated by the gateway. They can simply use these articles for information and guidance on their practice and work. However, and third, the gateway allows the users to perform further analysis of the selected articles, using techniques such as correlational analyses to identify patterns among the articles, and they can share the results further using social network platforms. The goal of these features is to allow users to customize their searches and to help them extract insights from the searches.

On the other hand, the Vidura chatbot is powered by Google Dialogflow, a natural language understanding (NLU) platform. Google Dialogflow was selected because it makes integration of user interface with mobile and web applications as well as interactive voice response systems and smart devices easy. Through analyzing users' queries, the chatbot identifies the intents of the users. Then when users type their questions as input to the chatbot, the chatbot matches the input and intents through a technique called intent classification. Intent classification can help the chatbot extract useful information for the users. In order to improve the chatbot performance, it employs variation inputs from the users to train the NLU model. 
To further improve the chatbot performance, the gateway will provide users with an online questionnaire upon signup to determine their domain proficiency (i.e., knowledge about COVID-19) and technology proficiency (i.e., experience with gateways in general). By categorizing the two proficiencies into high versus low, a two-by-two matrix can be created. Then, each diverse user can be put in one of the four quadrants (i.e., high in domain proficiency but low in technology proficiency; low in both domain and technology proficiencies). Then the chatbot will provide personalized responses according to individual users' position on the matrix, even if they ask the same question. This design is intentional, because as a wise advisor, Vidura is able to assess the baseline proficiency of a user, and determine the appropriate amount of guidance for each unique user. For example, Vidura will help a user placed in the quadrant defined by low proficiency in both domain and technology with domain guidance, even if the question is technical in nature. However, as the user improves in one or both proficiencies, their placement in the quadrant can shift (for more information about the Vidura chatbot, see Chandrashekara et al., 2018). The shifting is based on what Gibbs and colleagues (2021) explain as ML algorithms built into organizational decision-making in HMC research.

\section{Demonstrating the Chatbot's Role in Gateway Adoption}

Fortunati and A. P. Edwards (2020) argue that it is important to consider different methodologies to study HMC. We propose several mixed methods approaches to further study Vidura chatbot and identify its role in gateway adoption. First, it is important to conduct a market research study on Vidura. To do this, a series of interviews and focus groups can be conducted with clinicians and medical researchers combating COVID-19 at the front line, to better understand the challenges they face, their needs, routines, and their feedback on Vidura. Insights from the initial market research can further identify how the Vidura chatbot may meet the needs of the target clinicians and medical researchers.

Second, an experiment can be set up to compare the user outcomes between a control group and an experimental group. For example, user outcomes can include task completion time (start to end time of a search for COVID-19 literature) and success rates (able to find the desired COVID-19 literature on gateway or not) between a control group of users without the chatbot and the experimental group with the chatbot as the intervention. If there are statistically significant differences between the two groups, then the efficiency (shorter completion time in the experimental group) and effectiveness (higher success rates in the experimental group), then the relative advantages of the chatbot in helping clinicians and medical researchers find the COVID-19 literature they need can be demonstrated. Also, Lombard and $\mathrm{Xu}$ (2021) noted that voice (i.e., human-sounding versus machinesounding) can have an effect on users' HMC. Moreover, as machines are increasingly becoming human-like (Dehnert \& Leach, 2021), Davis and Stanovsek (2021) maintain that the use of an avatar can create a sense of character identity for the users, which can impact HMC. Therefore, different conditions of modality (text versus voice), voice (human- versus machine-sounding), and screen-based embodiment (no avatar versus with avatar) can serve as variables to compare the two stated outcomes in the experimental group. Additional user outcomes can also include satisfaction and likelihood to recommend the gateway with a 
chatbot to their peers, to further include variables related to diffusion and adoption of the KnowCOVID-19 gateway for patient care and medical research in combating the pandemic.

Third, a survey can also follow the experiment. The survey questionnaire can include variables such as perception of innovation attributes (Moore \& Benbasat, 1991) of the gateway and/or the chatbot, self-identified adopter categories (Noppers et al., 2015), opinion leadership/super-diffuser tendency (Boster et al., 2011), and demographic variables. Findings can help identify the demographic profiles of clinicians and medical researchers who are likely to be in favor of the gateway and/or chatbot. Then the developers can explore using the profiles to identify early adopters and opinion leaders to partner with, and pro-actively seek out input from the late majority and laggards to identify potential barriers to adoption, so the gateway and chatbot can be better designed for diffusion to more effectively and efficiently find COVID-19 literature to improve patient care, treatments, and preventions.

Fourth, the experiment can be video recorded, so the actual points-and-clicks by the clinicians and medical researchers can be analyzed by observational techniques to identify the usability of the gateway and chatbot. With video recordings, the analysis can focus on where these gateway users pause and take a long time to decide the next move. Similar analysis can also be performed to identify moments right before the users choose to engage with a chatbot, and how they continue with the gateway after receiving guidance and help from the chatbot. Such a study can also incorporate the talk out loud strategy, asking the users to verbally articulate their thought processes as they navigate the platform. Hence, the usability study can better relate the observation of navigation with the analysis of verbalized rationales, to help the developers better understand the workflow and routines of clinicians and medical researchers during the urgent time of combating COVID-19.

Fifth, eye-tracking techniques can be utilized to study where clinicians and medical researchers focus their eye gaze and visual attention on the KnowCOVID-19 gateway and the Vidura chatbot. Similar studies can be conducted to examine the relationships among visual attention/distraction to generate heat maps, and correlate the maps with other variables such as topic involvement and information recall (Gong et al., 2021), in addition to what HMC scholars have studied in utilizing eye-tracking software, such as how eye gaze signals active participation and intentionality in HMC (McEwen et al., 2020). The heat maps can also be used to correlate with findings from data from other studies, such as when users initiate a chat session with Vidura, and/or what questions are asked during the chat session.

Sixth, an open-ended interview with clinicians and medical researchers as participants immediately following the experiment can also solicit feedback on the design of gateway and chatbot for better user experience. Interviewing is particularly well-suited for gathering qualitative input. Furthermore, the analysis can also identify potential background factors (such as the thought processes, inherent assumptions, etc. of clinicians and medical researchers) that may have affected users' navigation of the gateway and engagement with the chatbot in both positive and negative ways. If a time gap allows for video processing, so the interview study is paired up with showing the video recording with talk out loud articulation, interviewers can better probe and ask the participant to recall and fully explain their experience with the gateway and chatbot, further triangulating different data sources and stimuli for a robust analysis. During this interview study, probes should be designed to gather a deep understanding of the work context of the clinicians and medical researchers during the pandemic, as such insights can help improve the contextual understanding of 
the COVID-19 pandemic. Interviews can also get at how users may have anthropomorphized the chatbot (Liu, 2021), attributing human characteristics to the machine.

Seventh, the verbatim dialogues between the users and the chatbot can be recorded and analyzed using conversational analysis techniques, to better understand how clinicians and medical researchers engage with the chatbot, and how to design responses for the chatbot to optimize the chat process with the least number of back-and-forth (i.e., adjacency pairs) between the users and the chatbot before successful task completion. We understand that clinicians and medical researchers are very busy, especially during the urgent time of combating the pandemic. Such a study can help developers identify the best way to design the optimal responses from the chatbot to the clinicians and medical researchers the technology is designed to serve. Also, this study can add to HMC research and examine selfdisclosure (Ling \& Björling, 2020), and this case, disclosure of questions about accomplishing certain tasks on KnowCOVID-19. Some clinicians and medical researchers may not ask certain questions to a human support personnel, but asking these questions to a machine advisor may be less embarrassing.

Finally, as the chatbot can be designed to gather user queries and intents, the data based on queries and intents can be further analyzed to identify clinicians and medical researchers with similar interests. Then the chatbot can allow these users to opt-in to be recommended and receive recommendations to connect with other users with similar interests, thus creating robust social networks that can lead to a robust user community of clinicians and medical researchers combating the COVID-19 pandemic worldwide. Social network analysis can be performed to identify weak ties, strong ties, and where potential ties turn into weak ties, and what factors may affect tie strengths and tie conversions across the world. This approach is important because HMC scholars have argued that machines can be mediators of social relationships (Fortunati \& Edwards, 2021), and social influence is a positive predictor of social machine adoption (Lutz \& Tamó-Larrieux, 2020).

\section{Conclusion and Implications}

The article set out to answer the research question: "How can an AI-powered chatbot help users, especially clinicians and medical researchers, effectively and efficiently identify reliable information with high evidence levels to help them make the best decisions at work?" We described a gateway called KnowCOVID-19 and the Vidura chatbot being prototyped to address this very question. The combination of the gateway and chatbot helps clinicians and medical researchers to overcome the big data problem, the quality problem, and the diffusion problem. What are the implications of this work?

Theoretically, this study advances diffusion of innovations theory (Rogers, 2003) by emphasizing the subsequent stage of sustainable implementation. Much diffusion research over-emphasizes the point of adoption, thus rendering sustainable implementation an oversight and under-studied area. This is understandable because traditional innovations studied in diffusion research are commercial products, and the researchers and companies behind are mainly concerned with the point of sales. In the case of gateways, our concern is beyond the point of initial adoption, to include meaningful use, widespread diffusion, and long-term sustainability in order to enable users to discover breakthrough science and research that help them better combat COVID-19. 
Second, much diffusion research has focused on opinion leadership and its impact on user adoption. However, less work has been done to investigate if and how helpful machines (i.e., chatbots) can support users in properly putting the innovation into practice during implementation. This article suggests that the adoption of chatbot with the gateway can help users sustain their adoption of the gateway, casting the chatbot and HMC as important for promoting and sustaining diffusion. Moreover, as chatbot becomes more commonly used in the society, human-chatbot interactions may in turn influence human-human interactions in recent HMC research (Gambino et al., 2020), thus changing how human opinion leaders may impact diffusion of innovations.

Third, studying the HMC between users and the chatbot in the use of KnowCOVID-19 represents an integration of actor-network theory (Latour, 2005) and diffusion theory (Rogers, 2003). Although humans and machines can be argued as ontologically different in HMC research (Guzman, 2020; Ling \& Björling, 2020), human agents (i.e., clinicians and medical researchers) and technical agent (i.e., the Vidura chatbot) can be argued as "potentially equivalent actors" (Banks \& de Graaf, 2020), hence their collaboration can be treated as leading to joint discoveries and identifications of research literature that can guide the best evidence-based health care, treatments, and preventions to combat the pandemic. While much of early diffusion research was built on quantitative and post-positivistic research, the case of KnowCOVID-19 and Vidura chatbot presents an opportunity for theoretical extension through a socio-material perspective.

Fourth, the chatbot can follow and track users' struggles with the gateway all the way until the point of discontinuation, if and when it happens. Much diffusion research focuses on successful adoption and sometimes implementation, perhaps due to the difficulty of finding adopters and/or discontinuers who opted-out to participate in research studies. This study suggests an opportunity to leverage chatbots to investigate discontinuation as an important decision point in diffusion research.

Practically, many gateway projects are federally funded for the initial development, not for long-term sustainability of the gateways. Additionally, there is usually no federal funding for technical support for users. As a result, implementation of user support is often an afterthought, and only recognized as a need when uptake increases notably, and the gateway begins to attract many new users beyond the inception projects. Voluntary user support by gateway developers when the technologies are in early diffusion cycles may be feasible. Once the gateway begins to gain traction in the user community, personalized user support becomes practically impossible. Scaling to keep up with adoption and guiding users' usage patterns becomes difficult in practice. The Vidura chatbot can solve this problem for gateway developers. While receiving support, guidance, and advice from the chatbot may appear less than ideal than human support in the early stages, with ML algorithms and more interaction data over time, Vidura may outperform human support. This is a possibility similar to the caution that HMC scholars Johanssen and Wang (2021) raised about how AI may ultimately surpass human intuition. This possibility is also aligned with the concern raised by HMC scholars Piercy and Gist-Mackey (2021), who warned about how automation may replace human workers in the future of work.

Furthermore, while the current focus is on literature as text-based data, the gateway can further automate data integration with electronic health records (EHR). In order to overcome the privacy concerns noted by HMC scholars (Lutz \& Tamó-Larrieux, 2020), this 
integration can be accomplished through privacy measures (Dinakarrao et al., 2019), such as using blockchain-based (Matos et al., 2018; Purohit et al., 2021) secure storage layer for data from EHR as value-based data analytics. As a result, future users can use the KnowCOVID-19 gateway to perform joint analysis with EHR data in concert with publication analytics research.

Moreover, the work on chatbot can also be extended to social robots (Banks et al., 2021), which has a three-dimensional embodiment and the ability to take in multiple sensory inputs, such as nonverbal cues, body temperature, and so forth from users. Social robots are also capable of conveying nonverbal cues to users, creating complex interactions to promote innovation adoption. Social robots can also be used in dangerous and/or hazardous environments, thus providing another means for protecting human agents from physical harms.

Finally, this article highlights the difference between innovations and diffusion, by putting the emphasis on the latter. While the KnowCOVID-19 gateway may be a good innovation, without the Vidura chatbot, adoption and diffusion over the long-term can be compromised. Similar to how vaccine for COVID-19 is an important innovation, vaccination is a separate science and is equally important for research attention. In other words, innovations cannot fully realize their societal impacts without the help of diffusion research. We argue that HMC, such as in the case of chatbots, can play an important role in the adoption of new technologies and other important innovation in the context of COVID-19. HMC insights can also be extended to the post-pandemic world and increase our preparedness for the next global crisis.

\section{Author Biographies}

Kerk F. Kee received his $\mathrm{PhD}$ in Organizational Communication, with an emphasis on workplace technologies, in the Department of Communication Studies at the University of Texas at Austin. He is currently an Associate Professor in the College of Media \& Communication at Texas Tech University. His research focuses on the diffusion of innovations in the scientific, health, and environmental contexts. His work has been funded by the Bill \& Melinda Gates Foundation, the Robert Wood Johnson Foundation, and US National Science Foundation (NSF), include an NSF CAREER award in 2015.

\section{https://orcid.org/0000-0002-0543-5009}

Prasad Calyam received his $\mathrm{MS}$ and $\mathrm{PhD}$ degrees from the Department of Electrical and Computer Engineering at The Ohio State University in 2002 and 2007, respectively. He is currently an Associate Professor in the Department of Electrical Engineering and Computer Science at University of Missouri-Columbia. His current research interests include distributed and cloud computing, computer networking, and cybersecurity. He is a Senior Member of IEEE. 
Hariharan Regunath received his MBBS and MD degrees from The Tamil Nadu Dr. MGR Medical University, India and Manipal University, India respectively. He received additional residency training in internal medicine and fellowship training in Infectious Diseases and Critical Care Medicine, both at University of Missouri-Columbia. He is currently an Assistant Professor of Clinical Medicine at the Department of Medicine Divisions of Pulmonary, Critical Care Medicine and Infectious Diseases at University of Missouri-Columbia. His current research interests include COVID-19, ARDS, Nosocomial Infections, Infective Endocarditis, Clinical Microbiology, CMV infections and Travel medicine.

https://orcid.org/0000-0003-4223-8765

\section{Acknowledgments}

This work is supported by the National Science Foundation under awards: OAC-1730655, OAC-2006816, and OAC-2007100. Any opinions, findings, and conclusions or recommendations expressed in this publication are those of the author(s) and do not necessarily reflect the views of the National Science Foundation.

\section{References}

Ackley, B., Ladwig, G., Swan, B., \& Tucker, S. (2008). Evidence based nursing care guidelines. Medical Surgical Interventions. Mosby Elsevier, syf, 15.

Ali, C. (2021, February 4). The 10 best chatbots of 2021. https://web.archive.org/ web/20210505133806/https://www.userlike.com/en/blog/best-chatbots

Baby, C. J., Khan, F. A., \& Swathi, J. (2017). Home automation using IoT and a chatbot using natural language processing. 2017 Innovations in Power and Advanced Computing Technologies (i-PACT).

Banks, J., \& de Graaf, M. (2020). Toward an agent-agnostic transmission model: Synthesizing anthropocentric and technocentric paradigms in communication. HumanMachine Communication, 1, 19-36. https://doi.org/10.30658/hmc.1.2

Banks, J., Koban, K., \& Chauveau, P. d. V. (2021). Forms and frames: Mind, morality, and trust in robots across prototypical interactions. Human-Machine Communication, 2, 81-103. https://doi.org/10.30658/hmc.2.4

Bharti, U., Bajaj, D., Batra, H., Lalit, S., Lalit, S., \& Gangwani, A. (2020, June 10-12).Medbot: Conversational artificial intelligence powered chatbot for delivering tele-health after COVID-19. 2020 5th International Conference on Communication and Electronics Systems (ICCES). https://doi.org/10.1109/ICCES48766.2020.9137944

Bieliauskas, S., \& Schreiber, A. (2017). A conversational user interface for software visualization. 2017 IEEE working conference on software visualization (vissoft). https://doi. org/10.1109/VISSOFT.2017.21

Boster, F. J., Kotowski, M. R., Andrews, K. R., \& Serota, K. (2011). Identifying influence: Development and validation of the connectivity, persuasiveness, and maven scales. Journal of Communication, 61, 178-196. https://doi.org/10.1111/j.1460-2466.2010.01531.x 
Calyam, P., \& Nair, S. (2020). OAC core: Small: Collaborative research: Conversational agents for supporting sustainable implementation and systemic diffusion of cyberinfrastructure and science gateways, NSF OAC \#2006816. https://nsf.gov/awardsearch/ showAward?AWD_ID $=2006816$

Chandrashekara, A. A., Talluri, R. K. M., Sivarathri, S. S., Mitra, R., Calyam, P., Kee, K., \& Nair, S. (2018). Fuzzy-based conversational recommender for data-intensive science gateway applications. 2018 IEEE International Conference on Big Data (Big Data). https:// doi.org/10.1109/BigData.2018.8622046

Davis, D. Z., \& Stanovsek, S. (2021). The machine as an extension of the body: When identity, immersion and interactive design serve as both resource and limitation for the disabled. Human-Machine Communication, 2, 121-135. https://doi.org/10.30658/hmc.2.6

Dehnert, M., \& Leach, R. B. (2021). Becoming human? Ableism and control in Detroit: Become human and the implications for human-machine communication. HumanMachine Communication, 2, 137-152. https://doi.org/10.30658/hmc.2.7

Dinakarrao, S. M. P., Sayadi, H., Makrani, H. M., Nowzari, C., Rafatirad, S., \& Homayoun, H. (2019). Lightweight node-level malware detection and network-level malware confinement in iot networks. 2019 Design, Automation \& Test in Europe Conference \& Exhibition. https://doi.org/10.23919/DATE.2019.8715057

Eren, M. E., Solovyev, N., Raff, E., Nicholas, C., \& Johnson, B. (2020). COVID-19 Kaggle Literature Organization. Proceedings of the ACM Symposium on Document Engineering 2020. https://doi.org/10.1145/3395027.3419591

Etzrodt, K., \& Engesser, S. (2021). Voice-based agents as personified things: Assimilation and accommodation as equilibration of doubt. Human-Machine Communication, 2, 57-79. https://doi.org/10.30658/hmc.2.3

Fast, E., Chen, B., Mendelsohn, J., Bassen, J., \& Bernstein, M. S. (2018). Iris: A conversational agent for complex tasks. Proceedings of the 2018 CHI Conference on Human Factors in Computing Systems. https://doi.org/10.1145/3173574.3174047

Fortunati, L., \& Edwards, A. (2021). Moving ahead with human-machine communication. Human-Machine Communication, 2, 7-28. https://doi.org/10.30658/hmc.2.1

Fortunati, L., \& Edwards, A. P. (2020). Opening space for theoretical, methodological, and empirical issues in human-machine communication. Human-Machine Communication, 1, 7-18. https://doi.org/10.30658/hmc.1.1

Gambino, A., Fox, J., \& Ratan, R. A. (2020). Building a stronger CASA: Extending the computers are social actors paradigm. Human-Machine Communication, 1, 71-86. https:// doi.org/10.30658/hmc.1.5

Gibbs, J. L., Kirkwood, G. L., Fang, C., \& Wilkenfeld, J. N. (2021). Negotiating agency and control: Theorizing human-machine communication from a structurational perspective. Human-Machine Communication, 2, 53-171. https://doi.org/10.30658/hmc.2.8

Gong, Z., Holiday, S., \& Cummins, R. G. (2021). Can't take my eyes off of the model: The impact of sexual appeal and product involvement on selective attention to advertisements. Journal of Marketing Theory and Practice, 2, 162-174. https://doi.org/10.1080/10 696679.2020.1803089

Greenhalgh, T. (2014). How to read a paper: The basics of evidence-based medicine: John Wiley \& Sons. 
Guzman, A. L. (2020). Ontological boundaries between humans and computers and the implications for human-machine communication. Human-Machine Communication, 1, 37-54. https://doi.org/10.30658/hmc. 1.3

Io, H., \& Lee, C. (2017). Chatbots and conversational agents: A bibliometric analysis. 2017 IEEE International Conference on Industrial Engineering and Engineering Management (IEEM). https://doi.org/10.1109/IEEM.2017.8289883

Johanssen, J., \& Wang, X. (2021). Artificial intuition in tech journalism on AI: Imagining the human subject. Human-Machine Communication, 2, 173-190. https://doi.org/10.30658/ hmc.2.9

John, R. J. L., Potti, N., \& Patel, J. M. (2017). Ava: From data to insights through conversations. Paper presented at 8th Biennial Conference on Innovative Data Systems Research (CIDR '17) January 8-11, 2017, Chaminade, California, USA.

Kee, K. F. (2020). OAC Core: Small: Collaborative research: Conversational agents for supporting sustainable implementation and systemic diffusion of cyberinfrastructure and science gateways, NSF OCA \#2007100. https://nsf.gov/awardsearch/showAward?AWD_ $\mathrm{ID}=2007100$

Kee, K. F., Le, B., \& Jitkajornwanich, K. (2021). If you build it, promote it, and they trust you, then they will come: Diffusion strategies for science gateways and cyberinfrastructure adoption to harness big data in the science, technology, engineering, and mathematics (STEM) community. Concurrency and Computation: Practice and Experience, e6192. https://doi.org/10.1002/cpe.6192

Kee, K. F., Sleiman, M., Williams, M., \& Stewart, D. (2016). The 10 attributes that drive adoption and diffusion of computational tools in e-science. In P. Navrátil, M. Dahan, D. Hart, A. Romanella, \& N. Sukhija (Eds.), 2016 XSEDE Conference: Diversity, Big Data, \& Science at Scale. New York: ACM. https://doi.org/10.1145/2949550.2949649

Latour, B. (2005). Reassembling the social: An introduction to actor-network-theory. Oxford University Press.

Ling, H. Y., \& Björling, E. A. (2020). Sharing stress with a robot: What would a robot say? Human-Machine Communication, 1, 133-158. https://doi.org/10.30658/hmc.1.8

Liu, J. (2021). Social robots as the bride? Understanding the construction of gender in a Japanese social robot product. Human-Machine Communication, 2, 105-120. https:// doi.org/10.30658/hmc.2.5

Lombard, M., \& Xu, K. (2021). Social responses to media technologies in the 21 st century: The media are social actors paradigm. Human-Machine Communication, 2, 29-55. https://doi.org/10.30658/hmc.2.2

Lutz, C., \& Tamó-Larrieux, A. (2020). The robot privacy paradox: Understanding how privacy concerns shape intentions to use social robots. Human-Machine Communication, 1, 87-111. https://doi.org/10.30658/hmc.1.6

Maniou, T. A., \& Veglis, A. (2020). Employing a chatbot for news dissemination during crisis: Design, implementation and evaluation. Future Internet, 12(7), 109. https://doi. org/10.3390/fi12070109

Martin, A., Nateqi, J., Gruarin, S., Munsch, N., Abdarahmane, I., Zobel, M., \& Knapp, B. (2020). An artificial intelligence-based first-line defence against COVID-19: Digitally screening citizens for risks via a chatbot. Scientific reports, 10, 19012. https:// doi.org/10.1038/s41598-020-75912-x 
Matos, D. R., Pardal, M. L., Adao, P., Silva, A. R., \& Correia, M. (2018). Securing electronic health records in the cloud. Proceedings of the 1st workshop on privacy by design in distributed systems. https://doi.org/10.1145/3195258.3195259

Mayer-Schönberger, V., \& Cukier, K. (2014). Big data: A revolution that will transform how we live, work, and think. Eanib Dolan/Mariner Books.

McEwen, R., Atcha, A., Lui, M., Shimaly, R., Maharaj, A., Ali, S., \& Carroll, S. (2020). Interlocutors and interactions: Examining the interactions between students with complex communication needs, teachers, and eye-gaze technology. Human-Machine Communication, 1, 113-131. https://doi.org/10.30658/hmc.1.7

Mehfooz F., Jha S., Singh S., Saini S., Sharma N. (2021) Medical chatbot for novel COVID19. In: Fong S., Dey N., Joshi A. (Eds.). ICT Analysis and Applications. Lecture Notes in Networks and Systems, vol 154. Springer, Singapore. https://doi.org/10.1007/978-98115-8354-4_42

Moore, G. C., \& Benbasat, I. (1991). Development of an instrument to measure the perceptions of adopting an information technology innovation. Information Systems Research, 2, 192-222. https://doi.org/10.1287/isre.2.3.192

Muslih, M., Supardi, D., Multipi, E., Nyaman, Y. M., \& Rismawan, A. (2018). Developing smart workspace based IOT with artificial intelligence using telegram chatbot. 2018 International Conference on Computing, Engineering, and Design (ICCED), 230-234. https://doi.org/10.1109/ICCED.2018.00052

Nair, S. S., \& Calyam, P. (2018). Experiences from a multi-disciplinary course sequence developmenton cyber and software automation in neuroscience. Paper presented at the Proceedings of Gateways 2018, Austin, TX.

NCBI, N. C. f. B. I., "PubMed”. (2020). https://pubmed.ncbi.nIm.nih.gov/

Ni L., Lu C., Liu N., Liu J. (2017) MANDY: Towards a smart primary care chatbot application. In: Chen J., Theeramunkong T., Supnithi T., Tang X. (Eds.). Knowledge and Systems Sciences. KSS 2017. Communications in Computer and Information Science, 780, 32-58. https://doi.org/10.1007/978-981-10-6989-5_4

NLM, N. L. o. M. (2020). LitCovid. https://www.ncbi.nlm.nih.gov/research/coronavirus/

Noppers, E. H., Keizer, K., Bockarjova, M., \& Steg, L. (2015). The adoption of sustainable innovations: The role of instrumental, environmental, and symbolic attributes for earlier and later adopters. Journal of Environmental Psychology, 44, 74-84. https://doi. org/10.1016/j.jenvp.2015.09.002

Oruche, R., Gundlapalli, V., Biswal, A. P., Calyam, P., Alarcon, M. L., Zhang, Y., Bhamidipati, N. R., Malladi, A., \& Regunath, H. (2021). Evidence-based recommender system for a COVID-19 publication analytics service. IEEE Access, 9, 79400-79415, 2021, https:// doi.org/10.1109/ACCESS.2021.3083583

Piercy, C. W., \& Gist-Mackey, A. N. (2021). Automation anxieties: Perceptions about technological automation and the future of pharmacy work. Human-Machine Communication, 2, 191-208. https://doi.org/10.30658/hmc.2.10

Prahl, A., \& Van Swol, L. (2021). Out with the humans, in with the machines?: Investigating the behavioral and psychological effects of replacing human advisors with a machine. Human-Machine Communication, 2, 209-234. https://doi.org/10.30658/hmc.2.11 
Purohit, S., Calyam, P., Alarcon, M. L., Bhamidipati, N. R., Mosa, A., \& Salah, K. (2021). HonestChain: Consortium blockchain for protected data sharing in health information systems. Peer-to-peer Networking and Applications, 1-17. https://doi.org/10.1007/ s12083-021-01153-y

Rodsawang, C., Thongkliang, P., Intawong, T., Sonong, A., Thitiwatthana, Y., \& Chottanapund, S. (2020). Designing a competent chatbot to counter the COVID-19 pandemic and empower risk communication in an emergency response system Outbreak, Surveillance, Investigation \& Response (OSIR) Journal, 13(2). http://osirjournal.net/index.php/ osir/article/view/193

Rogers, E. M. (2003). Diffusion of innovations (5th ed.). Free Press.

Russell, S., \& Norvig, P. (1995). Prentice Hall series in artificial intelligence: Prentice Hall.

SGCI, S. G. C. I. (2020). Science Gateways Community Institute. https://web.archive.org/ web/20201118141943/https://sciencegateways.org/

Szmigiera, M. (2021). Impact of the coronavirus pandemic on the global economyStatistics \& Facts. Statista.com. https://web.archive.org/web/20210808132753/https:// www.statista.com/topics/6139/covid-19-impact-on-the-global-economy/

Vekaria, K., Calyam, P., Sivarathri, S. S., Wang, S., Zhang, Y., Pandey, A., Chen, C., Xu, D., Joshi, T., \& Nair, S. (2020). Recommender-as-a-service with chatbot guided domainscience knowledge discovery in a science gateway. Concurrency and Computation: Practice and Experience, e6080. https://doi.org/10.1002/cpe.6080

VolppKevin, G. (2020). Asked and answered: Building a chatbot to address covid-19-related concerns. NEJM Catalyst Innovations in Care Delivery.

WHO, W. H. O. (2021). https://www.who.int/

Wilkins-Diehr, N. (2007). Science gateways-Common community interfaces to grid resources. Concurrency and Computation: Practice \& Experience, 19(6), 734-739.

Yoshikawa, Y., Kumazaki, H., \& Kato, T. A. (2021). Future perspectives of robot psychiatry: Can communication robots assist psychiatric evaluation in the COVID-19 pandemic era? Current Opinion in Psychiatry, 34(3), 277-286. https://doi.org/10.1097/ yco.0000000000000692

Zhang, Y., Calyam, P., Joshi, T., Nair, S., \& Xu, D. (2018). Domain-specific topic model for knowledge discovery through conversational agents in data intensive scientific communities. Paper presented at the 2018 IEEE International Conference on Big Data (Big Data). https://doi.org/10.1109/TKDE.2021.3093350 

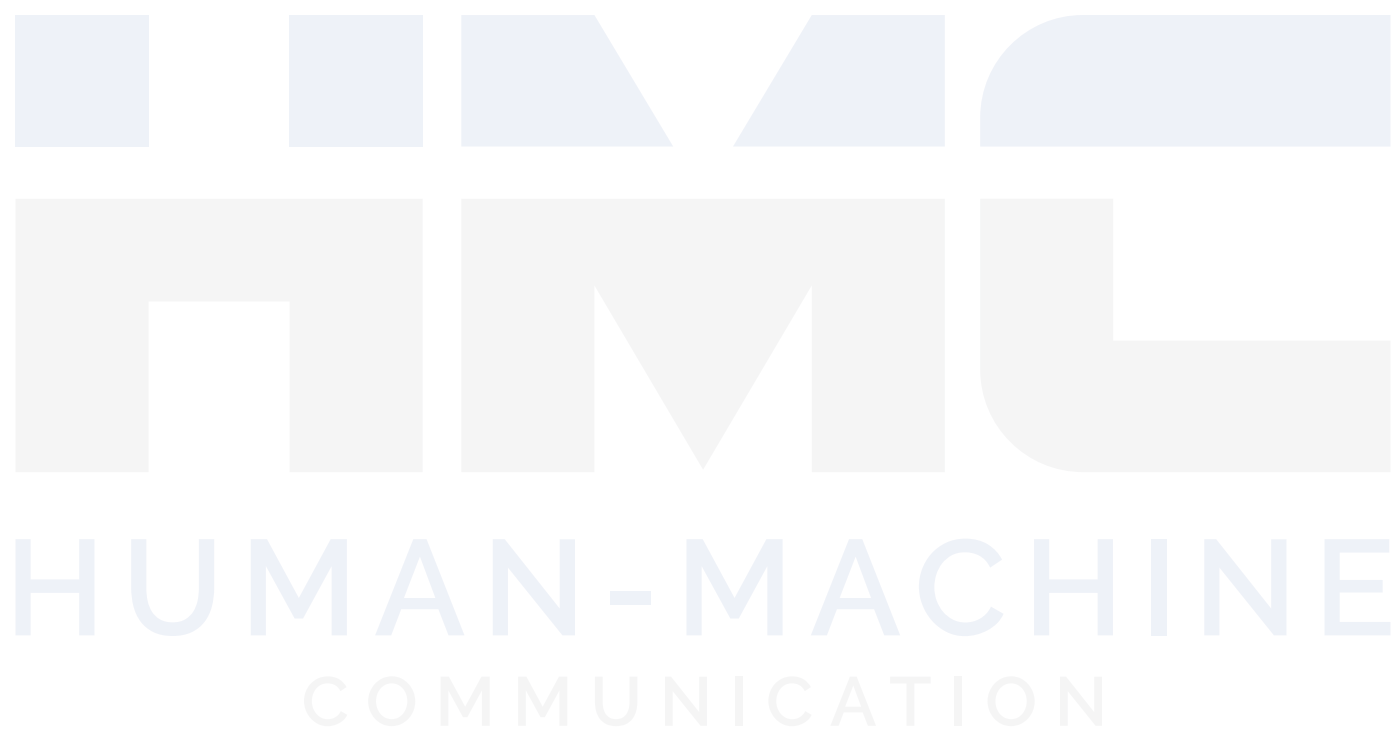